\title{
Antropologías y etnografías de los caminos. Introducción al dossier
}

\author{
Vladimir Caraballo Acuña \\ Candidato a doctor en Antropología Social de El Colegio de Michoacán (México). \\ Profesor de antropología de la Pontificia Universidad Javeriana (Bogotá). \\ $\checkmark$ caraballoa.m@javeriana.edu.co \\ (1) ORCID: 0000-0003-2736-6531 \\ ๑ Google Scholar \\ Daniel Ramírez Pérez \\ Candidato a doctor en Antropología Social de El Colegio de Michoacán (México). \\ $\checkmark$ ramirezp.daniel@gmail.com \\ (1) ORCID: 0000-0001-6883-8705 \\ a Google Scholar
}

Pensados como escenarios de trámite, como rutas que conectan dos puntos o simplemente como trasfondo, los caminos evocan una estabilidad tal que permiten darlos por sentado. Algo similar ocurre con el caminar, que puede ser entendido como un acto de locomoción, un paseo a bordo del propio cuerpo con el que se salva la distancia entre un origen y un destino. Sin embargo, las indagaciones antropológicas sobre la materialidad de las superficies y de los cálculos que las soportan - políticos, técnicos, económicos, etc.- han mostrado que los caminos, o específicamente las carreteras -roads-, no sólo son espacios de trámite entre dos lugares, sino infraestructuras con capacidad de producirlos (Dalakoglou y Harvey, 2012; Harvey, 2012; Harvey y Knox, 2015). De forma similar, en las últimas décadas han habido elaboraciones antropológicas sobre el caminar como un acto complejo en el que se conoce el mundo (Ingold, 2004, 2010); es decir, una práctica por medio de la cual se crea al caminante (Ingold, 2007; Ingold y Vergunst, 2008; Szakolczai y Horvath, 2018). Para nuestro interés, la producción del etnógrafo como un interlocutor pedestre (Hall y Smith, 2013, 2016; Pink, 2007, 2008; Smith, 2019) que afecta y es afectado por el contexto en el que trabaja. Algo que resulta capital si queremos tener presentes los cuestionamientos 
a las coordenadas espaciales (Ferguson y Gupta, 1997, 2008) y temporales (Fabian, 1983) con las que se ha entendido el "campo" de las prácticas etnográficas y de las antropologías (Krotz, 1993; Lins-Ribeiro y Escobar, 2008; Restrepo, 2012).

Nuestro interés por los caminos y las caminatas surgió de algunas reflexiones que sostuvimos con algunas compañeras de la cohorte 2016-2020 del Doctorado en antropología social de El Colegio de Michoacán. A principios del 2018, advertíamos que muchos de nosotros habíamos destinado una buena parte de nuestro trabajo de campo transitando carreteras, caminando por veredas en compañía de alguien más o, simplemente, tratando de identificar la continuidad de una trocha; coincidíamos, entonces, en que había que interrogar estas prácticas como parte del trabajo de campo. Con este objetivo diseñamos un seminario virtual que sostuvimos por un semestre; al final del mismo y con el ánimo de ampliar la perspectiva y de entablar diálogos con otros investigadores, propusimos el simposio antropología y etnografía de los caminos en el marco del XVII Congreso de Antropología en Colombia, germen de este dossier1. En este lapso, y gracias a los cuestionamientos que han provocado los distintos interlocutores con los que hemos tratado de poner en movimiento preguntas sobre los caminos, hemos pasado de una aproximación topográfica que los entiende como superficies de tránsito a una topológica, como plantea Harvey (2012) en la que podemos entenderlos como infraestructuras o, como planteamos aquí, como excesividad.

\section{Errantes}

Las discusiones sobre las relaciones de poder que producen y atraviesan la antropología como disciplina y a la etnografía como práctica (Da Col y Graeber, 2011; Ingold, 2017b; Krotz, 1993; Lins-Ribeiro y Escobar, 2008; Restrepo, 2006, 2012, entre otros), además de la aparición de propuestas para delimitarlas con relación a ciertas preocupaciones analíticas, nos llevaron a pensar que encontraríamos un cuerpo de elaboraciones bajo la rúbrica de antropologías o etnografías de los caminos. Suponíamos que algo tan "trivial" (cf. Szakolczai y Horvath, 2018) o mecánico (cf. Ingold, 2007) como caminar, o tan habitual como los caminos, habría llamado la atención de la disciplina y que, entonces, podríamos encontrar algo semejante a lo que ya reconocíamos como "antropología de la minería" (Ferry, 2009)

Queremos reconocer a nuestras compañeras Carmen Moreno y Adriana Alas por las discusiones que sostuvimos en el seminario. También queremos agradecer a Penny Harvey y a Simón Uribe por sus comentarios durante el simposio y por su participación en este dossier; igualmente a Luís Alberto Suárez Guava y a Laura Guzmán por el apoyo para la concreción de este proyecto editorial. 
-o de los minerales preciosos, como aparecería con posterioridad (Ferry, Vallard y Walsh, 2020)-, "de la memoria" (Severi, 2010) o "del turismo" (Salazar, 2006, 2017a). Para nuestra sorpresa, y de forma similar a lo que anota Laura Guzmán en su artículo para este número, no tuvimos éxito en las búsquedas que realizamos; no encontramos tal cosa como una antropología de los caminos y menos una sobre los caminantes ${ }^{2}$. Salvo algunas excepciones, en la literatura antropológica en español, los caminos tienen una marcada presencia metafórica; en inglés los resultados son otros ${ }^{3}$. De allí que nuestra aproximación esté influenciada por la bibliografía en inglés y particularmente por aquella ligada a las discusiones sobre la (in)movilidad, por ejemplo el número especial de Social Anthropology que editó Noel Salazar (2017b); a la antropología de las infraestructuras que han propuesto Penny Harvey y Hannah Knox (2015); a la posibilidad del camino como etnografía de Dimitris Dalakoglou y la misma Harvey (Dalakoglou y Harvey, 2012); al análisis etnográfico del caminar que proponen Ingold y Vergunst (2008); o a la atención etnográfica a las materialidades de los caminos que han servido de soporte para las investigaciones de Richard Kernaghan (2012), Simón Uribe (2017) o Dimitris Dalakoglou (2010).

La falta de un cuerpo más o menos definido que permitiera ahondar en el tema fue lo que nos llevó a construir nuestra propia ruta; una vía de tres carriles con la que pretendíamos abarcar asuntos teóricos, etnográficos y literarios - una clasificación que todavía nos incomoda-. Por la senda teórica, uno de los guías era Walter Benjamin (2005), en particular por sus elaboraciones sobre el paseante -flâneur- parisino del siglo XIX, una figura muy ilustrativa para pensar la ciudad mercancía y a quien deambulaba exhibiéndose como anuncio por ella, pero que nos dejaba fuera de lugar porque nuestras inquietudes estaban ligadas a ámbitos rurales, a caminatas por veredas o a sendas que la vegetación ha cubierto. Similares dificultades experimentábamos cuando tratábamos de pensar las trochas, los atajos, los "desechos", los caminos o las carreteras en locaciones no fronterizas o, cuando menos, como infraestructuras

\footnotetext{
No queremos desconocer la existencia de investigaciones dedicadas a estos temas, más bien lo que resaltamos es su dispersión y la dificultad que ello reviste para pensar en un interés disciplinario. Tal vez lo más cercano a esto sea el la propuesta para una antropología del caminar — anthropology of walkingde Szakolczai y Horvath (2018); sin embargo, ésta es más bien una indagación etnoarqueológica sobre el sedentarismo y las implicaciones de esto para la modernidad.

Dentro de la literatura que encontramos hay acercamientos al caminar como práctica identitaria y de producción de conocimiento quinésico para los Rarámuri de México (Lares, 2015; Martínez-Ramírez, 2007) y para los Anlo-Ewe en Ghana (Geurts, 2002); otros ejemplos en esta "línea" se pueden encontrar referidos en Tim Ingold (2007, esp. cap. 3). También encontramos algunas reflexiones sobre prácticas etnográficas que tienen como base las caminatas y las relaciones con animales en procesos de cacería o ecoturismo (Hurn, 2012) o en paseos citadinos con perros (Lane, 2015), por citar algunos ejemplos.
}

Brian Larkin (2013) enlista una buena parte de estos trabajos en inglés. 
de conectividad pedestre y no motorizada como sí lo son la carretera - motorway - que sirve de límite entre Albania y Grecia (Dalakoglou, 2010), la autopista - highway- que conecta el Perú con el Ecuador (Kernaghan, 2012) o la carretera -road- que permite la movilidad entre los departamentos de Nariño y Putumayo (Uribe, 2017). Asumimos, entonces, que la opción era errar; avanzamos un tanto por la vía de las (in)movilidades pensadas desde la antropología (Clifford, 1997) y la sociología (Rojek y Urry, 1997). Como turistas - una figura incómoda pero cada vez más recurrente para la antropología (Graburn, 2017; Leite, Castañeda y Adams, 2019; Salazar, 2017a) - avanzamos por una ruta demarcada pero extraña, con actores poco familiares y en otro idioma. Esto último resultó muy provocador, porque al tratar de traducir las nociones asociadas a aquellas infraestructuras - motorway, highway, road, dams- notamos que la diferencia lingüística también sugería tamaños, materiales, usos, experticias, ritmos de construcción, velocidades de flujo y, como mostraremos más adelante, excesividad ${ }^{4}$.

El recorrido nos develó una relación "evestigial", nos mostró las huellas de las propias pisadas (Haber, 2011) ${ }^{5}$. Nos evidenció la miopía que había en el visualizar los caminos sólo como superficies sobre las que transitan cosas o personas, como puntos que conectan destinos, parafraseando a Ingold (2007). La errancia nos llevó a considerar la excesividad, nos puso sobre un pasaje por el que ya habíamos transitado, nos dejó ad portas de lo que podría ser una entrada del "Onceno Tomo" de la primera enciclopedia de Tlön, ese mundo lleno de paradojas y cuya geometría, según describe Borges - quien estaba en nuestro carril literario-, "declara que el hombre que se desplaza modifica las formas que lo circundan" (Borges, 2002); y agregaríamos: así como éstas lo modifican a él.

\section{Banalidades controversiales}

Ingold ha equiparado el caminar con el hablar y el pensar (Ingold, 2004, 2010; Ingold y Vergunst, 2008) y ha dicho que estas son actividades habituales que tienen como característica el hecho de que acontecen en un pliegue particular de comienzos y finales que responden siempre a la presencia de otros, en una relación diferida, no necesariamente in situ y

\footnotetext{
Más que palabras, entonces, lo que encontramos fueron conceptos (Trouillot, 2011) y esa es la razón por la cual sostenemos aquí los términos en inglés. Una tarea pendiente, pero ya iniciada por algunas de las autoras que incluimos en este dossier, es empezar a pensar en la especificidad de los caminos, las trochas, las veredas o de las mismas fronteras; en entender lo que implica caminar para los interlocutores y los alcances que esto tiene como parte del trabajo etnográfico.

Alejandro Haber (2011) describe a la relación que hay entre la huella y su negativo, la planta que la realizó, como algo evestigial. Es decir, una evidencia inmediata que está diferida en el tiempo y en el espacio. La investigación, como arguye él, avanza sobre vestigios pero también deja huellas.
} 
contingente. Dalakoglu y Harvey (2012), por su parte, han sostenido que los caminos, en apariencia mundanos, son lugares controvertidos porque prometen y/o retan la conectividad, la movilidad, el crecimiento o la circulación y porque, como infraestructuras, entrecruzan escalas. Esto, anotan estos autores, permite que las etnografías puedan atender a relaciones entre entidades y, a la vez, navegar a través de categorías habituales de pensamiento: "de la materialidad a los sujetos humanos, del Estado a la sociedad y de ahí a los individuos; de las empresas capitalistas globales a las comunidades locales; [entre otros]" (Dalakoglou y Harvey, 2012, p. 460. Nuestra traducción).

Si como sostiene Johanes Fabian la antropología es una práctica que se desarrolla en eventos y movimientos, su especificidad no está dada por un único discurso -digamos el de la antropología de los caminos, que seguimos buscando- sino por el hecho de tener que confrontar un mundo que no es el de las antropologías (Fabian, 2008, p. 351). Por eso mismo, como ha indicado en otra parte, la teoría importa y la etnografía es una forma de hacer esto, ya que es una práctica comunicativa que ocurre, se mueve, confronta y por eso permite que otros también se muevan a medida que uno mismo se revela afectado por ellos (Fabian, 2001). Esta es una apuesta muy similar a la que plantea Giovanni Da Col (2017) en relación con que la teoría etnográfica tiene por principio y fin la incertidumbre y la no correspondencia; su apuesta contrasta explícitamente con la advertencia que hace Ingold sobre la confusión de la antropología con la etnografía o, lo que sería lo mismo, la teorización con la descripción (Ingold, 2017a, 2017b).

A pesar de haber bautizado al simposio que tuvimos en el XVII Congreso de Antropología en Colombia como antropología y etnografía de los caminos, y de que para este dossier de la RASV tratamos de ampliar la discusión integrando plurales, el recorrido que hemos sostenido hasta la redacción de esta introducción nos ha llevado a pensar que lo que estamos tratando de provocar son prácticas etnográficas sobre esas entidades banales y controversiales que son los caminos y, más específicamente, sobre su excesividad. Creemos que estas etnografías, múltiples, disimiles y diferencialmente ubicadas en el "establecimiento antropológico" (Restrepo, 2012) deben estar en la capacidad de dialogar con otras prácticas disciplinarias y, como lo recuerdan varias autoras en este número, con otros conocimientos. Esto resulta crucial porque casi todos somos caminantes y en ese sentido habría posibilidad para la igualdad gnoseológica que supone el "todos somos etnógrafos" del que habla Luis Reygadas (2014) y resulta fundamental para un ejercicio 
dialógico de la antropología -sobre las interacciones en el trabajo de campo- (Yeh en este número).

Creemos que los caminantes, los caminos o sus caminatas no son entidades de poca valía para las "antropologías del sur" (Krotz, 1993) o las "subalternizadas" o "disidentes" (Restrepo, 2012) que tienen como locación de producción, o de interés, América Latina: porque son muchas las carreteras y trechos que recorren los migrantes; porque muchas veces la actividad política institucional avanza sobre "elefantes blancos" de concreto; o, sencillamente, porque para las poblaciones subalternizadas marchar es parte del repertorio de protesta; en fin, porque son entidades excesivas que pueden desbordar la práctica antropológica y aproximarnos a otros mundos.

\section{La excesividad de los caminos y de los caminares}

La palabra "exceder" viene del latín excedere, compuesto de ex (hacia el exterior) y cedere (ceder, ir, marchar, caminar). Partiendo de lo anterior, los artículos de este dossier nos ayudan a pensar un asunto específico, pero muy amplio y productivo, acerca de los caminos, el caminar y, más ampliamente, de las infraestructuras: su excesividad. Utilizamos esta palabra, un poco rara, para hablar del exceso no como un sustantivo, ni de lo excesivo como un simple adjetivo, sino de la excesividad como una cualidad en sí misma, por derecho propio. Y aquí estamos pensando en la excesividad como una cualidad de distintas entidades que, en permanente construcción, suelen desbordar, retar, cuestionar, parámetros de interpretación usuales (Bataille, 1974; Gandolfo, 2013; Gandolfo y Ochoa, 2017) y que nos llevan a comprender las teorías que las personas mismas construyen de sus mundos (Suárez-Guava, 2013) a partir de las formas de caminar y de lo que cuentan acerca de ellas. La excesividad de los caminos es construida a partir de las experiencias de muy distintas entidades, de las relaciones entre ellas y de espacio-temporalidades que exceden la planeación y son siempre imprevisibles. Esta excesividad de eventos, entidades, experiencias, suele escapar a los compromisos analíticos de la academia; la realidad, dicen los autores de la introducción al Crumpled Paper Boat, siempre está impregnada de algo más, "de algo intangible, evanescente, resistente a la descomposición analítica" (Pandian y McLean, 2017, p. 19. Nuestra traducción)

La excesividad de los caminos y del caminar que se relata en los artículos de este dossier nos ayuda a pensar la ambigüedad de las infraestructuras mismas, constituidas en una suerte de suspensión (Gupta, 2018) paradójica (Howe et al., 2016) entre la ruina y la promesa (Appel, Anand y Gupta, 2018; Velho y Ureta, 2019), entre lo móvil y lo 
detenido, entre la solidez y la liquidez. Los caminos y caminares aquí relatados están lejos de la planeación y la proyección, lejos de su concepción como conexiones seguras y lineales entre puntos, entre orígenes y destinos. Algo anti-productivo, anti-económico es creado en las relaciones que construyen a los caminos. Anti-productivo no por destructivo sino por estar constituido por la sorpresa permanente, por el asombro, por lo impredecible, por lo que no puede ser del todo controlado, por lo que no obedece a la voluntad de alguien sino a extrañas combinaciones entre entidades. Como bellamente lo dice Chaustre en su artículo, caminar es "dar vueltas en un ir y venir sin ir para ningún lado". Los caminos de este dossier siempre están en construcción, son ambiguos e inciertos. No hay, entonces, una diferencia entre el camino como infraestructura y el caminar como actividad desarrollada en ella. No parece haber, para decirlo en relación con la ya clásica propuesta de Brian Larkin, mayor diferencia entre la materia que garantiza la movilidad y la materia que se mueve a través de ella (Larkin, 2013). No hay caminos prediseñados así como no hay caminantes cuya existencia sea previa al caminar: el camino no existe sin actividad de caminar, de transitar; no hay camino sin las huellas de los antepasados; las marcas corporales del caminar, son el camino; el sonido, los olores, el tacto, son el camino (Ingold, 2004, 2010); las palabras intercambiadas por los caminantes, son el camino.

\section{Entidades y encuentros inciertos}

Por esto, en lo narrado por las autoras nos encontramos con entidades muy distintas. Los caminos y el caminar no emergen de la voluntad de ellas como si se tratara de unidades discretas, sino de sus encuentros que, también, son encuentros excesivos ${ }^{6}$ : el hormigón peruano, como cuenta Penny Harvey, emerge como un material a la vez sólido y maleable de la relación entre agua, arena, temperatura, abandono y cuidado; el agua robada por un extraño debe ser transportada garantizando que construya su propio camino hasta el lugar en donde será sembrada nuevamente, como cuenta Anahí Chaparro para la Alta Amazonía peruana; el faldón, las rocas, las mulas y los espíritus del norte del Tolima tienen fuerza y se articulan en el caminar de los sembradores de café en el texto de Laura Guzmán. Así, por ejemplo, cuenta Guzmán que los cultivadores la alentaban a que le "buscara la forma" para no caerse en el faldón, con el coco de café colgando de la cintura, y sintiendo la fuerza que la jalaba hacia adelante; "buscarle la forma", como le indicaban los campesinos, delata en ellos una teoría particular sobre la relación entre estas entidades, el

Partiendo de la teoría cuántica, Karen Barad ha desarrollado muy bellas provocaciones acerca de la indeterminación como característica de distintas entidades, del espacio y del tiempo (Barad, 2010). 
caminar y los caminos: "buscar la forma" de caminar implica la renuncia a la imposición planeada de la voluntad, a la imposición de un proyecto que, nuevamente, produzca algo como ir de un lugar a otro. Al contrario, esta teoría está constituida por una negociación permanente y nunca controlable en su totalidad. Los caminos, el caminar y los caminantes emergen de la relación entre las entidades en lugar de constituirse como identidades fijas y discernibles. Es a estas relaciones irreducibles al control, y a los caminos como infraestructuras siempre impredecibles y en proceso de construcción, a lo que nos referimos cuando hablamos de su excesividad.

Estas entidades dan forma a los caminos a través de lo que unas se dan a las otras. A la manera de Munn (Munn, 1986) las cualidades de distintas entidades son transferidas a los caminantes y a sus caminares de distintas maneras. Se trata de relaciones de afectación mutua (Weszkalnys, 2016) en las que, nuevamente, no hay separaciones claras entre entidades activas y pasivas, entidades que se mueven gracias a otras que se mantienen inertes y que solemos naturalizar como "infraestructuras". El maíz, las hormigas, los osos y los zorros, por ejemplo, les dan fuerza a los caminantes, igual que la serpiente fueteadora y el licor les da el don de la velocidad; confeccionar vasijas de cerámica mientras los niños están pequeños puede demorar su aprendizaje del caminar pero, una vez han aprendido, unos golpecitos con arcilla en las plantas de los pies puede dotarlos de fuerza (Chaparro); del "cuidado" de los pobladores locales de Perú, según los ingenieros, depende la relación entre la maleza, el agua y las cualidades del asfalto de las carreteras (Harvey); "poner los pies al través" le permite al cultivador de café quitarle fuerza al faldón (Guzmán); los intercambios lingüísticos y numéricos ambiguos y a veces indescifrables entre los checadores, ruteros o controladores de Tijuana, crean una temporalidad también abierta en medio de la movilidad capitalista de la frontera (Yeh); mientras tanto, los intercambios de alimentos entre humanos y animales dan forma y son formados por los caminares de unos y otros (Chaparro).

Y hay aquí un interesante contraste entre los intercambios analizados por Yeh y las analizadas por Chaustre: si en el primer caso se trata de (no)relaciones cercanas a "dones puros" opuestos a intercambios recíprocos, en los términos de Derrida (1995) -ver también Siegel (2013)-, en el segundo es la reciprocidad entre, por ejemplo, animales y humanos a lo largo de sus travesías, lo que sustenta las relaciones; si en el primer

\footnotetext{
Aunque los estudios infraestructurales suelen ocuparse de infraestructuras convencionales (tuberías, puentes, carreteras), autores como Paul Kockelman (2013, entre otros) o Atsuro Morita (2017) han querido avanzar en procesos o dinámicas infraestructurales más amplias que avancen en pensar "infraestructuralmente" en entidades distintas: desde ecuaciones y algoritmos, hasta el arroz y otros componentes del suelo (ver también, Caraballo Acuña, 2017).
} 
caso es la fuerza de una suerte de anti-economía comunicativa, ambigua, a veces indescifrable, la que sostiene las relaciones en "la ruta", en el caso de Chaustre es la certeza de lo que se intercambia y de la existencia misma de los intercambios lo que las garantiza. En todos los casos, sin embargo, lo que se "dan" unas entidades a otras no es un intercambio calculado sino... otra cosa: algo que excede el cálculo, la proyección, y que es siempre abierto, impredecible. Lo que estos intercambios habilitan es un conjunto de temporalidades que supera las narrativas lineales de la modernidad.

\section{Ritmos en lugar de progreso}

Aunque las infraestructuras suelen estar relacionadas con narrativas de progreso y modernización como la misma Penny Harvey muestra en su artículo, producen poéticamente experiencias temporales muy distintas, contradictorias y, como hemos venido diciendo, en permanente construcción. Los análisis aquí presentados nos ayudan a relativizar la suposición de la movilidad como único ritmo del capitalismo en el cual las infraestructuras (los caminos entre ellas) cumplirían un papel central. Al lado de ello, los artículos nos invitan a pensar en las pausas, en las detenciones, en las suspensiones como constituyentes de un moteado de experiencias temporales que, unidas con materialidades específicas, son usadas por las personas de manera creativa con objetivos distintos: acelerar el caminar, ralentizarlo, volverlo incierto, hacerlo suave o duro, etcétera (una etnografía particularmente sensible al respecto es la de Kernaghan, 2012, en Perú). Si bien la emergencia de los estudios sobre infraestructuras ha tenido lugar en el marco de preguntas acerca del movimiento en el mundo contemporáneo, distintos autores han mostrado que se trata de entidades ambiguas (Howe et al., 2016) que habilitan tanto el movimiento como la detención, que mueven gracias a la fijación, que fijan gracias al movimiento, que se mueven y se detienen ellas mismas. En esta ambigüedad propia de las infraestructuras cobran un papel central los "ritmos" alrededor suyo (Tsing, 2015). Se trata de pausas, disminuciones de velocidad, aceleraciones, suspensiones, etcétera, superpuestas unas sobre otras. La pregunta sobre las infraestructuras ha llevado, paradójicamente, dicen los editores de The promises of Infrastructures citando a Laura Bear, a pensar en la lentitud de la aceleración:

La atención rigurosa a la infraestructura [nos permite] ver demoras, acreción, suspensión, reparación, resistencia y reutilización. La atención etnográfica a la infraestructura puede 'socavar en última instancia cualquier idea de que la economía de la velocidad o del tiempo -la simplificación más grave de la lógica de la eficienciaes el corazón del capitalismo. En cambio, podemos explorar las 
formas heterogéneas de duración, espera, pausa, obsolescencia y retraso que también caracterizan sus ritmos generativos'. (Bear, 2015 como se citó en Appel, Anand y Gupta, 2018, p. 16. Nuestra traducción)

El artículo de Rihan Yeh es particularmente sensible acerca de estos ritmos alrededor de la movilidad vial en Tijuana. La autora muestra la relación entre la construcción temporal que hacen los ruteros y sus cuentas, la experiencia temporal de la movilidad propia de la frontera México-Estados Unidos y las experiencias temporales construidas entre ella, como etnógrafa, y uno de esos ruteros. Son esas distintas experiencias temporales las que toman forma por medio de una comunicación siempre ambigua entre los ruteros y entre uno de ellos y la etnógrafa. En lugar de un tiempo que se acumula y va hacia adelante, se trata más bien del diálogo de distintas construcciones temporales que, como nos enseñara hace tanto Mijaíl Bajtín (1975), implican espacialidades también alejadas de las geometrías, las superficies planas, los telones de fondo.

\section{Espacialidades}

Por último, también las experiencias espaciales alrededor de los caminos y del caminar exceden la planeación. Como dijimos al inicio, la espacialidad de los caminares y caminos que componen este dossier está lejos de reducirse a una función técnica que conecta orígenes y destinos. Pensando más en la función poética a la que se refiere Larkin siguiendo a Roman Jakobson (Larkin, 2013), la configuración espacial de los caminos es construida también a partir de métricas, resonancias, encuentros inesperados entre las entidades humanas y no humanas. Lejos de la planeación, estas resonancias construidas en el aquí y ahora constituyen espacios más parecidos a los rizomas descritos por Deleuze y Guattari (2004) y bellamente ejemplificados en los análisis de Laura Ogden acerca de las interacciones en los pantanos californianos. Allí, la autora describe los pantanos como paisajes de exceso rizomático constituidos por redes confusas y no lineales, en un productivo espacio "en medio" de las cosas y en un permanente devenir. Los paisajes descritos, dice Ogden, "son ensamblajes constituidos por humanos y no humanos, procesos materiales y semióticos, historias reales y parcialmente recordadas. Más aún, estas diversas propiedades forman un ensamblaje caracterizado por heterogeneidades y conexiones rizomáticas" (Ogden, 2011, p. 35).

La excesividad de los caminos y caminares reunidos aquí está más allá incluso de la constitución semiótica del espacio que relaciona signos y objetos y que haría parte de la poética infraestructural. Laura Guzmán por ejemplo, se refiere a las "geometrías esquivas" que forman la espacialidad 
de los recolectores de café, unas geometrías que, justamente, esquivan las coordenadas fijas, las líneas y la previsión; en este carácter esquivo de la construcción espacial, los cultivadores deben "encontrarle la forma" a su relación con la peligrosa inclinación del faldón, con la fuerza de la gravedad, con el material del piso, con los sonidos que también constituyen a los caminos; pero, más allá de ese control, por ejemplo, siempre hay algo, impredecible, excesivo, que no puede ser controlado: los espíritus que habitan los caminos o las rocas que pueden subir en lugar de bajar. Los campesinos saben relacionar signos y objetos, saben construir relaciones de representación, pero también saben habitar la impredecibilidad de los espacios. La espacialidad de los caminos se llena entonces de encanto, de misterio, de exceso. Quizá el ejemplo más literal sea la naturaleza particularmente espacial de los duendes que el taita Arturo Jacanamejoy Mavisoy le contaba a Laura Chaustre: los duendes que habitan los caminos tienen la capacidad de "voltear el mundo", de crear nuevos caminos, de hacerse escuchar lejos cuando están cerca y cerca cuando están lejos, de dejar huellas de niño que señalan la dirección inversa a la que en realidad se mueven, de hacerle trenzas a los caballos mientras los refunde, de refundir las herramientas de trabajo, de confundir a las personas, de hacer que se pierdan.

Es esta lógica incierta impuesta por los duendes del piedemonte amazónico en el sur de Colombia la que resume la naturaleza de los caminos y caminares de este dossier. En los caminos que veremos las palabras son ambiguas, los materiales son duros y maleables al mismo tiempo, los espíritus hacen regalos, pero también confunden. Estos caminos superan las lógicas económicas de la producción y nos ayudan a pensar otro tipo de relaciones. En ellos los signos no están del todo dados, la interpretación no es siempre sencilla, no es sencillo saber qué representa qué. La invitación que queremos hacer es parecida a la que hace Kathleen Stewart para pensar "un lugar al lado del camino": la región minera de los Apalaches del suroeste de Virginia occidental, dice Stewart, es un lugar que insiste en la necesidad de las brechas en el significado de los signos para, desde ellas, crear la posibilidad de la historia. Allí surge un espacio narrativo prolífico, que interrumpe la búsqueda de la esencia de las cosas y de las rápidas conclusiones con una poética de aplazamiento y desplazamiento, con un reencuentro rumiante en la particularidad de las formas y epistemologías locales. Este es el espacio de las brechas, cuyos signos se vuelven luminosos en la búsqueda de sus significados esquivos pero palpables (Stewart, 1996, pp. 3-4). Para Stewart, estas epistemologías constituyen un "lugar al lado del camino". Seguimos su sensible etnografía; sólo que, en lugar de tratarse de lugares "al lado del camino", los nuestros están "en el centro" (en caso de que hubiera tal cosa) de ellos. 


\section{Los artículos}

El dossier inicia con la traducción de un artículo de Penny Harvey, publicado originalmente en 2010 y ahora traducido por nosotros con el título: "Cimentando relaciones: La materialidad de las carreteras y los espacios públicos en el Perú provincial". Su artículo - parte de una amplia e inspiradora obra sobre caminos, infraestructuras y materialidad (Dalakoglou y Harvey, 2012; Harvey y Knox, 2015; Harvey, Krohn-Hansen y Nustad, 2019; Harvey, Morita y Jensen, 2016)-, nos sitúa de inmediato en las preocupaciones centrales del dossier. Harvey desarrolla un sensible análisis de lo que llama "la seducción del concreto" y las estéticas del poder estatal en Perú. Para ello, transita etnográficamente entre varios casos en los que el hormigón ha sido usado, particularmente, en Ocongate al sur del país. La autora explica esta seducción del hormigón a partir de una ambigüedad material (entre sólido y maleable) que se proyecta políticamente a las tensiones irresueltas entre la estandarización y la experimentación, la fijación y el deterioro, la promesa y la espera. A partir de distintos casos (carreteras, plazas, monumentos, edificios) la autora demuestra cómo esta ambigüedad material y política ha tenido un papel fundamental como componente estético del proceso de modernización, descentralización y formación de élites locales en la región.

Si este artículo nos permite hacer preguntas políticas sobre la materialidad del hormigón, en "Anacleto. El tiempo y el don en una ruta del transporte público (Tijuana, México)", Rihan Yeh nos orienta hacia otra materialidad: la de las interacciones comunicativas que subyacen al transporte público en un escenario típicamente fronterizo. Entre los checadores o ruteros mexicanos que controlan los tiempos de los buses, esta materialidad es, también, ambigua, fugaz y delicadamente sostenida por actos de habla poco predecibles cuyos significados suelen no ser claros: chistes, insinuaciones, ambigüedades. Yeh aprovecha estas interacciones comunicativas para mostrar, a la luz de la idea del "don puro" de Jacques Derrida (1995), opuesta a la de "don" de Marcel Mauss que aparece en los artículos de Guzmán y de Chaparro, cómo a través de ellas se articulan distintos tipos de tiempo frente a la movilidad que cabría esperar en un escenario de capitalismo fronterizo. Acorde con el espíritu del dossier, la autora aprovecha sus reflexiones sobre el tiempo en la ruta para invitarnos a reflexionar acerca de los intercambios etnográficos que realizamos con quienes colaboran, o no, con nuestros trabajos. Los caminos y las prácticas que les dan forma, resultan útiles aquí para hacer preguntas más amplias acerca del ejercicio etnográfico. 
También aprovechando los caminos para realizar una reflexión acerca de la labor etnográfica, el texto de Laura Guzmán, "Buscar la forma: ir sometiéndose y andar toriando caminos en el Norte del Tolima, Colombia", es fundamental para pensar en la complejidad del espacio en la configuración de estas infraestructuras. A través de una sensible descripción de su aprendizaje con los cultivadores de café y de maíz en el Tolima colombiano, Guzmán nos presenta lo que uno de ellos le describiera como "buscar la forma" de hacer las cosas: una suerte de articulación no planeada entre las "fuerzas" de distintas entidades (la falda de la montaña, los pies del cultivador, los granos de maíz o de café, el viento, los sonidos, las piedras, las mulas) que, aunque en manos del cultivador que camina, supera cualquier tipo de planeación y se muestra siempre impredecible. El caminar que nos muestra Guzmán está lejos de aquellos caminares contemplativos que encontramos, por ejemplo, en los bonitos ensayos de Robert Louis Stevenson y William Hazlitt (2018) o en el de David Le Breton (2014). "Buscar la forma", nos muestra la autora, es un tipo de práctica que hace explícita la existencia del espacio no como un telón de fondo neutral y dispuesto para la contemplación, sino como el producto de relaciones de mutua afectación, de reciprocidad, entre entidades muy distintas.

Laura Chaustre nos pone frente a las huellas de los ancestros, bien o mal fallecidos, en el piedemonte amazónico del sur de Colombia. La autora nos ayuda a pensar nuevamente el tiempo que ya veíamos en Yeh: si en su artículo se trata de tiempos que se otorgan, se superponen, se consumen, en Chaustre son tiempos de un permanente ir y volver: "caminar", dice, "es hacer memoria de los mayores al ir y volver, al volver a ir y devolverse". La autora nos muestra cómo el tiempo del pueblo indígena Camëntsa es un tiempo hecho cuerpo ("en los callos está el tiempo", dice) y construido por la reciprocidad que se opone al tiempo del progreso y a la extracción de recursos que toma sin devolver. Se trata de la circularidad de un tiempo relacionada con la circularidad de alimentos y seres que otorgan cualidades a los caminantes: agua que quita la fuerza, chicha que la da, lugares que son pesados y confunden al caminante, culebras que dan velocidad. Al mismo tiempo, la excesividad del espacio toma una forma privilegiada aquí en los duendes de los que ya hemos hablado, duendes que son "presencias" que están y no están al mismo tiempo, que dan vuelta al mundo y a sus coordenadas: lo lejos se vuelve cerca y el ir se convierte en venir. Los duendes, como entidades que resumen buena parte de los sentires de este dossier, refunden las herramientas de trabajo; es decir, refundiéndolas, refunden la productividad y, con ella, los principios de planeación hechos de fines en lugar de principios, de planes en lugar de improvisación y de encuentros siempre inciertos. 
El dossier cierra con "Territorio habitado y territorio como derecho: reflexiones desde el caminar Kichwa Lamista" en la Alta Amazonía peruana, de Anahí Chaparro. En su artículo la autora nos muestra cómo el territorio Kichua Lamista ha estado históricamente construido a partir del encuentro de distintos caminos que van desde aquellos trazados por los indígenas durante el "boom del caucho", hasta los de los militares, los narcotraficantes, los grupos subversivos, los espíritus, la sal, el agua y los astros. La autora contrapone el caminar indígena basado en los intercambios entre seres (personas, animales o plantas entre otros) con la reciente vigilancia satelital, la construcción de la carretera Fernando Belaúnde Terry y la legislación ambiental que, definiendo áreas de conservación con restricciones para los pobladores, han implicado distintos conflictos que Chaparro nos propone leer a partir del caminar y de los caminos. De esta manera, la autora nos brinda un amplio espectro de caminares y de caminos que se mueven, de un lado, entre el baile, los matrimonios, la caza, la crianza, el parentesco y las fiestas y, de otro, entre la protección ambiental, la vigilancia satelital, el comercio y las relaciones políticas alrededor de las titulaciones de tierra. En su recorrido, Chaparro pone sobre la mesa territorios que, además de ser objeto de conflictos políticos, son construidos y constructores de cuerpos, de sensaciones y encuentros que, nuevamente, se parecen más a rizomas que a fronteras, y que superan distinciones clásicas entre lo cultural y lo natural, entre lo humano y lo no humano, entre lo vivo y lo muerto.

\section{Referencias bibliográficas}

Appel, H., Anand, N. y Gupta, A. (eds.). (2018). Introduction: Temporality, Politics, and the Promise of Infrastructure. En The promise of infrastructure (pp. 1-38). Durham: Duke University Press.

Bajtín, M. (1975). Teoría y estética de la novela. Madrid: Taurus.

Barad, K. (2010). Quantum Entanglements and Hauntological Relations of Inheritance: Dis/continuities, SpaceTime Enfoldings, and Justice-to-Come. Derrida Today, 3(2), 240-268.

Bataille, G. (1974). Obras Escogidas. Barcelona: Seix Barral.

Bear, L. (2015). Navigating austerity: Currents of debt along a South Asian river. Stanford, CA: Stanford University Press.

Bear, L., Ho, K., Tsing, A. y Yanagisako, S. (2015). Gens: A feminist manifesto for the study of capitalism. Theorizing the con temporary, Cultural Anthropology website. Recuperado de https://culanth.org/fieldsights/652-gens-a-feminist-manifesto-for-the-studyof-capitalism.

Benjamin, W. (2005). El Flâneur. En Libro de los pasajes (pp. 421-457). Madrid: Ediciones Akal S.A. 
Borges, J.L. (2002). Tlön, Uqbar, Orbis Tertius. En Ficciones (pp. 16-35). Madrid: Alianza Editorial.

Caraballo Acuña, V. (2017). De cómo etnografiar ontologías e infraestructuras: una reseña sobre Paul Kockelman (2016). The Chicken and the Quetzal. Incommensurate Ontologies and Portable Values in Guatemala's Cloud Forest. Durham: Duke University Press, 208 pp. Revista Antípoda (sección en línea).

Clifford, J. (1997). Routes. Travel and Translation in the late Twentieth Century. Cambridge: Harvard University Press.

Da Col, G. (2017). Two or Three Things I Know About Ethnographic Theory. HAU: Journal of Ethnographic Theory, 11(1), 1-8. DOI: http://dx.doi.org/10.14318/hau7.1.002

Da Col, G. y Graeber, D. (2011). Foreword: The return of ethnographic theory. HAU: Journal of Ethnographic Theory, 1(1), 6-35. DOI: https://doi.org/10.14318/hau1.1.001

Dalakoglou, D. (2010). The Road: An ethnography of the Albanian-Greek cross-border motorway. American Ethnologist, 37(1), 132-149. DOI: https://doi.org/10.1111/j.15481425.2009.01246.x

Dalakoglou, D. y Harvey, P. (2012). Roads and Anthropology: Ethnographic Perspectives on Space, Time and (Im)Mobility. Mobilities, 7(4), 459-465. DOI: https://doi.org/10.1080 $/ 17450101.2012 .718426$

Deleuze, G. y Guattari, F. (2004). Mil mesetas. Capitalismo y esquizofrenia. Valencia: Pre-Textos.

Derrida, J. (1995). Dar (el) tiempo. Barcelona: Paidós.

Fabian, J. (1983). Time and the Other: How Anthropology Makes its Object. New York: Columbia University Press.

Fabian, J. (2001). Anthropology with an Attitude: Critical Essays. Stanford: Stanford University Press.

Fabian, J. (2008). Antropologías del mundo. Interrogantes. En G. Lins-Ribeiro y A. Escobar (eds.), Antropologías del mundo. Transformaciones disciplinarias dentro de sistemas de poder (pp. 335-353). Popayán: The Wenner-Gren Foundation, Ciesas, Envión.

Ferguson, J. y Gupta, A. (1997). Discipline and Practice: "The Field" as Site, Method, and Location in Anthropology. En J. Ferguson y A. Gupta (eds.), Anthropological Locations: Boundaries and Grounds of a Field Science (pp.1-46). Berkeley y Los Angeles: University of California Press. DOI: https://doi.org/10.1525/9780520342392-002

Ferguson, J. y Gupta, A. (2008). Más allá de la "cultura": Espacio, identidad y las políticas de la diferencia. Antípoda, (7), 233-256.

Ferry, E.E. (2009). Asuntos de consumo: sobre el lugar del consumo en la antropología de la minería. Relaciones, 30(118), 57-95.

Ferry, E.E., Vallard, A. y Walsh, A. (eds.). (2020). The Anthropology of Precious Minerals. Toronto: University of Toronto Press.

Gandolfo, D. (2013). Formless: A day at Lima's Office of Formalization. Cultural Anthropology, 28(2), 278-298. DOI: https://doi.org/10.1111/cuan.12004

Gandolfo, D. y Ochoa, T.R. (2017). Ethnographic excess. En A. Pandian y S. McLean (eds.), Crumpled Paper Boat. Experiments in ethnographic writing (pp. 185-188). Durham: Duke University Press. 
Geurts, K.L. (2002). On Rocks, Walks, and Talks In West Africa: Cultural Categories and an Anthropology of the Senses. Ethos, 30(3), 178-198.

Graburn, N. (2017). Key Figure of Mobility: the tourist. Social Anthropology, 25(1), 83-96. DOI: https://doi.org/10.1111/1469-8676.12394

Gupta, A. (2018). The future in ruins. Thoughts on the temporality of infrastructures. En H. Appel, A. Gupta, y N. Anand (eds.), The promise of infrastructures (pp. 62-79). Durham: Duke University Press.

Haber, A. (2011). Nometodología Payanesa: Notas de metodología indisciplinada (con comentarios de Henry Tantalean, Francisco Gil García y Dante Angelo). Revista Chilena de Antropología, (23), 9-49. DOI: https://doi.org/10.5354/0719-1472.2011.15564

Hall, T. y Smith, R.J. (2013). Stop and Go: A Field Study of Pedestrian Practice, Immobility and Urban Outreach Work. Mobilities, 8(2), 272-292. DOI: https://doi.org/10.1080/17450 101.2012.659470

Hall, T. y Smith, R.J. (2016). Pedestrian circulations: urban ethnography, the mobilities paradigm and outreach work. Mobilities, 11(4), 498-508. DOI: https://doi.org/10.108 0/17450101.2016.1211819

Harvey, P. (2012). The Topological Quality of Infrastructural Relation: An Ethnographic Approach. Theory, Culture y Society, 29(4/5), 76-92. DOI: https://doi.org/10.1177/ 0263276412448827

Harvey, P. y Knox, H. (2015). Roads: An Anthropology of Infrastructure and Expertise. Ithaca: Cornell University Press.

Harvey, P., Krohn-Hansen, C. y Nustad, K.G. (eds.). (2019). Anthropos and the material. Durham: Duke University Press.

Harvey, P., Morita, A. y Jensen, B.C. (2016). Introduction: Infrastructural complications. En P. Harvey, A. Morita, y C.J. Bruun (eds.), Infrastructures and social complexity. A companion (pp. 1-22). Londres: Routledge.

Howe, C., Lockrem, J., Appel, H., Hackett, E., Boyer, D., Hall, R., ... y Ballestero, A. (2016). Paradoxical Infrastructures: Ruins, Retrofit, and Risk. Science Technology and Human Values, 41(3), 547-565. DOI: https://doi.org/10.1177/0162243915620017

Hurn, S. (2012). Humans and Other Animals: Cross-Cultural Perspectives on Human-Animal Interactions. London: Pluto Press.

Ingold, T. (2004). Culture on the Ground: The World Perceived Through the Feet. Journal of Material Culture, 9(3), 315-340. DOI: https://doi.org/10.1177/1359183504046896

Ingold, T. (2007). Lines: A brief history. London \& New York: Routledge, Taylor y Francis Group. DOI: https://doi.org/10.4324/9781315625324

Ingold, T. (2010). Footprints through the weather-world: walking, breathing, knowing. Journal of the Royal Anthropological Institute, (16), S121-S139. DOI: https://doi.org/10.1111/ j.1467-9655.2010.01613.x

Ingold, T. (2017a). iSuficiente con la etnografía! Revista Colombiana de Antropología, 53(2),143159. https://www.redalyc.org/articulo.oa?id=1050/105052402007

Ingold, T. (2017b). Anthropology contra ethnography. HAU: Journal of Ethnographic Theory, 7(1), 21-26. DOI: https://doi.org/10.14318/hau7.1.005 
Ingold, T. y Vergunst, J.L. (2008). Introduction. En T. Ingold y J.L Vergunst (eds.), Ways of Walking. Ethnography and Practice on Foot (pp. 1-19). Hamphshire: Ashgate Publishing Ltd.

Kernaghan, R. (2012). Furrows and Walls, or the Legal Topography of a Frontier Road in Peru. Mobilities, 7(4), 501-520. DOI: https://doi.org/10.1080/17450101.2012.718932

Kockelman, P. (2013). The anthropology of an equation. Sieves, spam filters, agentive algorithms, and ontologies of transformation. HAU: Journal of Ethnographic Theory, 3(3), 33-61.

Krotz, E. (1993). La producción de la antropología en el Sur: características, perspectivas, interrogantes. Alteridades, 3(6), 5-11.

Lane, K.L. (2015). Canine Connections: fieldwork with a dog as research assistant. Anthropology in Action, 22(3), 27-38. DOI: https://doi.org/https://doi.org/10.3167/aia.2015.220304

Lares, A.F. (2015). Caminos Rarámuri para sostener o acabar el mundo. Teoría etnográfica, cambio climático y antropoceno. Mana, 26(1), 1-35.

Larkin, B. (2013). The Politics and Poetics of Infrastructure. Annual Review of Anthropology, 42, 327-343. DOI: https://doi.org/10.1146/annurev-anthro-092412-155522

Le Breton, D. (2014). Caminar. Elogio de los caminos y de la lentitud. Buenos Aires: Waldhuter.

Leite, N., Castañeda, Q. y Adams, K. (eds.). (2019). The Ethnography of Tourism. Edward Bruner and Beyond. London: Lexington Books.

Lins-Ribeiro, G. y Escobar, A. (eds.). (2008). Antropologías del mundo. Transformaciones disciplinarias dentro de sistemas de poder. Popayán: The Wenner-Gren Foundation, Ciesas, Envión.

Martínez-Ramírez, M.I. (2007). La composición de la persona en el pensamiento rarámuri. Revista pueblos y fronteras digital, 4, 1-21.

Morita, A. (2017). Multispecies Infrastructure: Infrastructural Inversion and Involutionary Entanglements in the Chao Phraya Delta, Thailand. Ethnos, 82(4), 738-757.

Munn, N.D. (1986). The Fame of Gawa: A Symbolic Study of Value Transformation in a Massim (Papua New Guinea) Society. Durham: Duke University Press.

Ogden, L. (2011). Swamplife. People, gators, and mangroves entangled in the Everglades. Minneapolis: University of Minnesota Press.

Pandian, A. y McLean, S. (eds.). (2017). Crumpled paper boat. Experiments in ethnographic writing. Durham \& London: Duke University Press.

Pink, S. (2007). Walking with video. Visual Studies, 22(3), 240-252. DOI: https://doi. org/10.1080/14725860701657142

Pink, S. (2008). An urban tour: The sensory sociality of ethnographic place-making. Ethnography, 9(2), 175-196. DOI: https://doi.org/10.1177/1466138108089467

Restrepo, E. (2006). Diferencia, hegemonía y disciplinación en antropología. Universidad humanistica, (62), 43-70.

Restrepo, E. (2012). Antropologías disidentes. Cuadernos de Antropología Social, 35, 55-69.

Reygadas, L. (2014). Todos somos etnógrafos. Igualdad y poder en la construcción del conocimiento antropológico. En C. Oehmichen Bazán (ed.), La etnografía y el trabajo de en las ciencias sociales (pp. 91-118). Ciudad de México: Universidad Nacional Autónoma de México. 
Rojek, C. y Urry, J. (eds.). (1997). Touring Cultures. Transformatios of Travel and Theory. New York: Routledge, Taylor \& Francis Group.

Salazar, N.B. (2006). Antropología del turismo en países en desarrollo: análisis crítico de las culturas, poderes e identidades generados por el turismo. Tabula Rasa, (5), 99-128.

Salazar, N.B. (2017a). Anthropologies of Tourism: What's in a Name? American Anthropologist, 119(4), 723-725. DOI: https://doi.org/10.1111/aman.12954

Salazar, N.B. (2017b). Key figures of mobility: an introduction. Social Anthropology, 25(1), 5-12. DOI: https://doi.org/10.1111/1469-8676.12393

Severi, C. (2010). El sendero y la voz. Una antropología de la memoria. Buenos Aires: Editorial $\mathrm{Sb}$.

Siegel, J. (2013). False beggars: Marcel Mauss, the gift, and its commentators. Diacritics, 41(2), 60-79. DOI: https://doi.org/10.1353/dia.2013.0010

Smith, R.J. (2019). Visually available order, categorization practices, and perceptionin-action: a running commentary. Visual Studies, 34(1), 28-40. DOI: https://doi. org/10.1080/1472586X.2019.1622445

Stevenson, R.L. y Hazlitt, W. (2018). Caminar. Madrid: Nórdica Libros.

Stewart, K. (1996). A Space on the Side of the Road: Cultural Poetics in an "Other" America. Princeton: Princeton University Press.

Suárez Guava, L.A. (2013). Guacas. Teorías del mundo en los Andes colombianos. Mopa Mopa, $1(22), 10-49$.

Szakolczai, A. y Horvath, A. (2018). Walking into the Void. A Historical Sociology and Political Anthropology of Walking. London: Routledge.

Trouillot, M.R. (2011). Adieu, cultura: surge un nuevo deber. En Transformaciones globales. La antropología y el mundo moderno. Cali: Universidad del Cauca, Ceso-Universidad de los Andes.

Tsing, A. (2015). The Mushroom at the End of the World: On the Possibility of Life in Capitalist Ruins. Princeton: Princeton University Press.

Uribe, S. (2017). Frontier Road. Power, History, and the Everyday State in the Colombian Amazon. Oxford: John Wiley y Sons Ltd.

Velho, R. y Ureta, S. (2019). Frail modernities: Latin American infrastructures between repair and ruination. Tapuya: Latin American Science, Technology and Society, 2(1), 428-441. DOI: https://doi.org/10.1080/25729861.2019.1678920

Weszkalnys, G. (2016). A doubtful hope: resource affect in a future oil economy. Journal of the Royal Anthropological Institute, 22(1), 127-146 